\title{
O TRIPLO DESAFIO DA \\ INOVATIVIDADE: O IMPACTO DO PRECONCEITO
}

\section{THE TRIPLE CHALLENGE OF INNOVATIVENESS: THE IMPACT OF PREJUDICE}

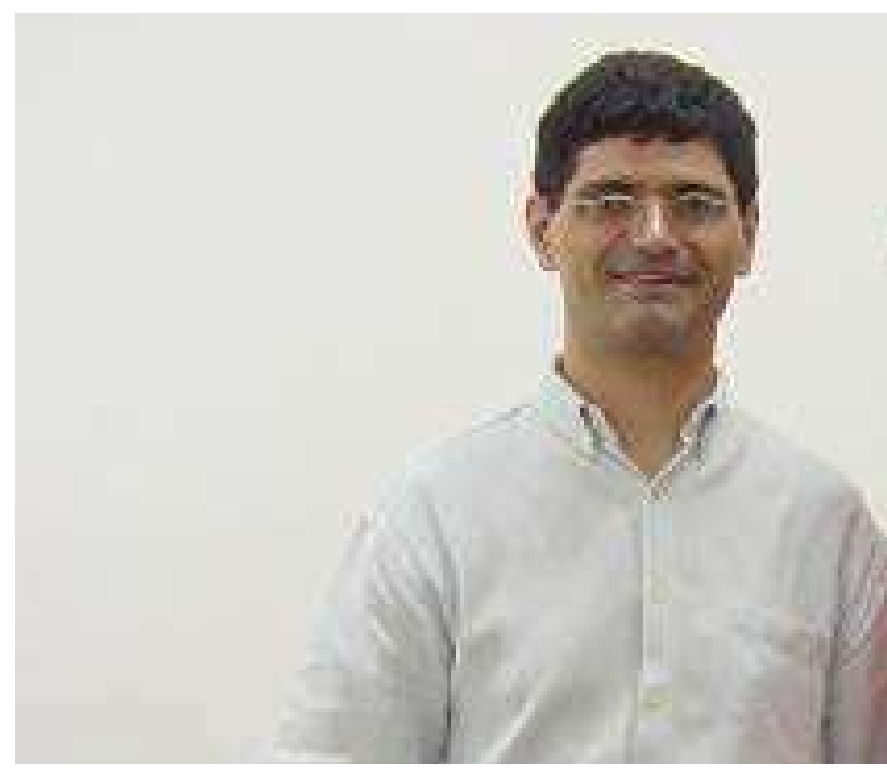

\section{Gregorio Varvakis}

Doutor em Manufacturing Engineering pela

Loughborough University of Technology, Inglaterra. Professor titular do Departamento de Engenharia e Gestão do Conhecimento da Universidade Federal de Santa Catarina.

g.varvakis@ufsc.br

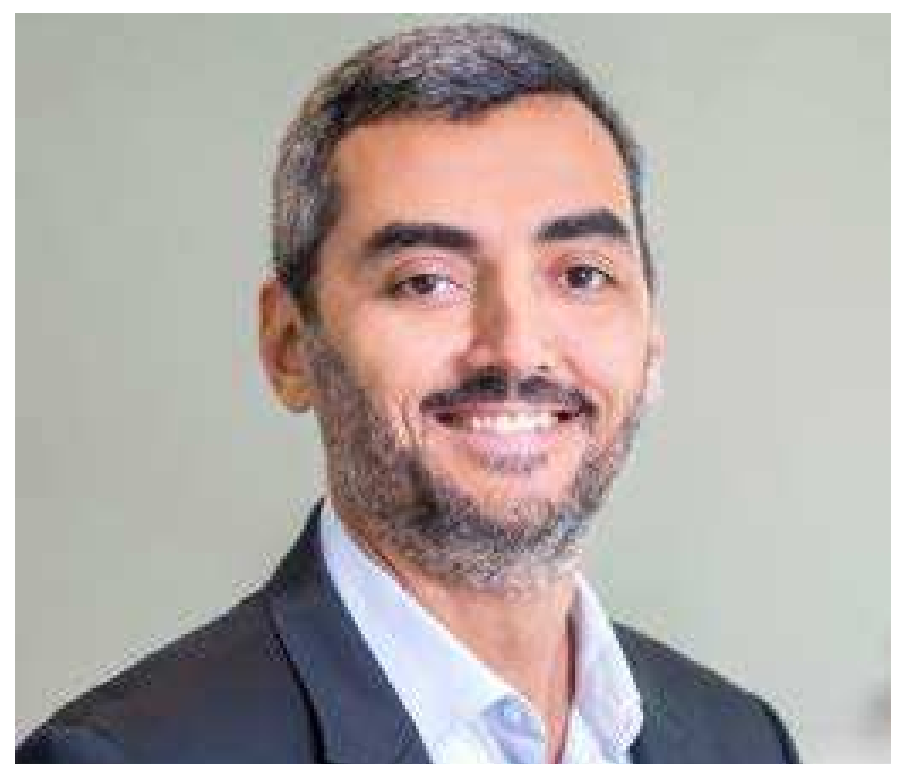

Maurício Manhães

Doutor em Engenharia e Gestão do

Conhecimento pela Universidade Federal de Santa Catarina, Brasil.

Professor do Departamento de Design da Savannah College of Art and Design, EUA mmanhaes@scad.edu 


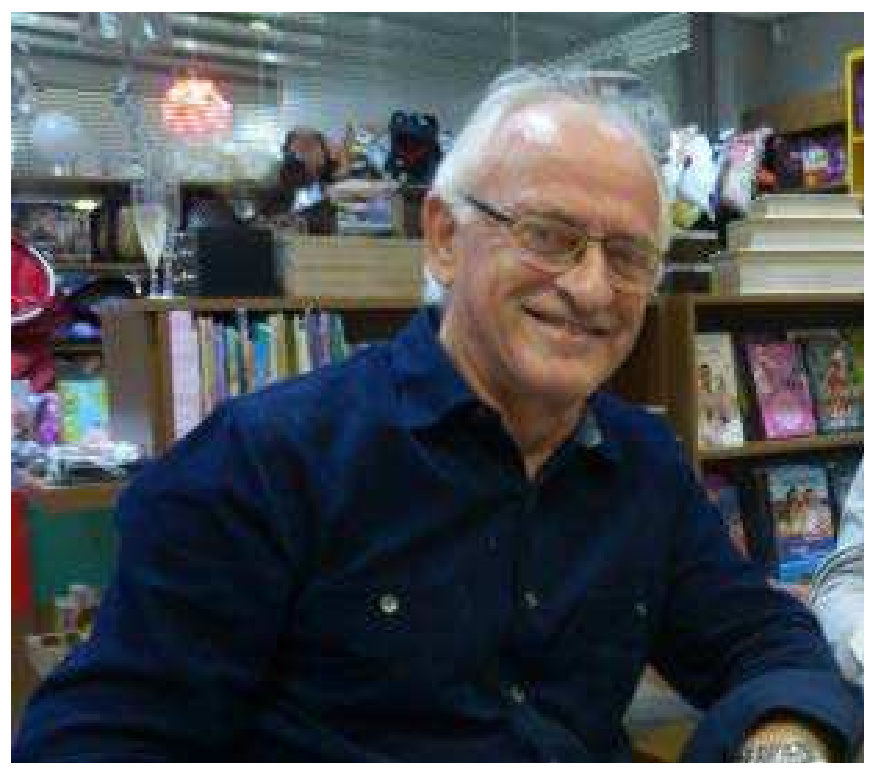

Tarcisio Vanzin

Doutor em Engenharia de Produção pela

Universidade Federal de Santa Catarina, Brasil.

Professor voluntário do Programa de Pós

Graduação em Arquitetura e Urbanismo e do

Programa de Pós Graduação em Engenharia e

Gestão do Conhecimento da Universidade Federal

de Santa Catarina

tvanzin@gmail.com

\section{RESUMO}

O interesse organizacional contemporâneo a respeito da inovação levou a várias tentativas de domá-la por meio de amplas chamadas para as práticas de criatividade e design. Na maioria das vezes, essas chamadas fogem do confronto entre o processo de renovação contínua do efêmero de um lado; e a tradição e o preconceito, por outro. $\mathrm{O}$ objetivo deste texto é fazer sentido de um discurso para aumentar o potencial de criação de conhecimento de grupos, de modo a atuarem na direção do futuro, para um melhor desempenho e longevidade. Baseado no conceito de Necessidade de Enquadramento (Need for Closure), a partir de uma perspectiva hermenêutica e inspirado por uma abordagem metodológica reflexiva, o presente estudo lança luz sobre os impactos do preconceito nos esforços inovadores de grupos. Assim, este estudo propõe uma heurística baseada em determinantes de inovatividade relacionados a preconceito (denominada Prejudice Related Innovativeness Determinants Heuristic - PRIDHe), para aumentar efetivamente o potencial inovativo de grupos sociais. A heurística sugere formas de alocar pessoas em e define uma política de governança para grupos, a fim de proporcionar um ambiente criativo onde o preconceito não somente limita as ações como sugere novas oportunidades de atuar em direção ao futuro. A principal contribuição teórica deste trabalho reside nas reflexões sobre os impactos positivos do preconceito nos esforços inovativos. Em seu núcleo, o discurso proposto neste texto pode ser resumido como: organizações cientes de seus preconceitos possuem maior probabilidade de apresentar um melhor desempenho. 


\section{PALAVRAS-CHAVE}

Grupos. Necessidade de Enquadramento. Inovação. Conhecimento. Design. Sensemaking. Preconceito. Hermenêutica.

\section{ABSTRACT}

The contemporary organizational interest about innovation has led to several attempts to tame it through broad calls for creativity and design practices. Most of the times, these calls evade the confrontation between the process of continuous renewal of the ephemeral on one side; and the tradition and prejudice on the other. The purpose of this study is to make sense of a discourse that augment the potential of groups to create knowledge so to act into the future, towards better performance and longevity. Based on the concept of Need for Closure, from a hermeneutic perspective and inspired by a reflexive methodological approach, the present study sheds light on the impacts of prejudice on innovative efforts of groups. Supported by an empirical study and quantitative data analysis, it proposes a Prejudice Related Innovativeness Determinants Heuristic (PRIDHe) to enable groups to effectively augment their innovative potential. The heuristic suggests forms of assigning people to and defines a governance policy for groups, in order to provide a creative environment where prejudice does not so much confine actions as suggest new opportunities to act into the future. The main theoretical contribution of this work lies in the reflections about the positive impacts of prejudice in innovative efforts. The discourse proposed by this text can be summarized as: organizations that are aware about their prejudices and the impacts of these are more likely to perform better.

\section{KEYWORDS}

Groups. Need for Closure. Innovation. Knowledge. Design. Sensemaking. Prejudice. Hermeneutic.

\section{INTRODUÇÃO}

O interesse organizacional a respeito da inovação levou nas últimas duas décadas a diversas tentativas de controlar práticas de criatividade e design (AMABILE, 1996; BUCHANAN, 2015; JOHANSSON-SKÖLDBERG; WOODILLA; ÇETINKAYA, 2013). Na maioria das vezes, estas tentativas apresentam um ponto cego representado pelo confronto entre o esforço de um grupo para a criação de algo inovador de um lado; e a tradição e o preconceito desse mesmo grupo de outro (GINOULHIAC, 2009).

Diversos estudos acadêmicos apontam para a incompatibilidade entre a adoção de métodos e a obtenção de proposições originais (THOMPSON, 1997; VERGANTI; ÖBERG, 2013); no caso organizacional, proposições inovadoras. A base de todo método reside na capacidade de prever, de reduzir incertezas, de permitir a repetição (GADAMER, 2004), elementos que são contrários aos requisitos fundamentais de toda inovação 
(ADORNO, 1965; SCHUMPETER, 1927; SIMONTON, 2010). A esta, espera-se que seja original, surpreendente e, por isso mesmo, lucrativa.

Sendo assim, a inovação entendida como o resultado de um processo "não-organizado" (SCHUMPETER, 1927; SEWELL, 2008) coloca um desafio significativo para as organizações, justamente focadas em "organizar." Ou seja, a solução para organizar ou organizacionalisar a inovação deve ser estabelecida em um meta-nível, que permita um equilíbrio ideal entre os inerentes esforços organizacionais de manutenção (TANGEN, 2005) e a necessária originalidade dos esforços organizacionais de inovação.

Conforme ressaltado acima, estudos acadêmicos desencorajam a adoção de métodos lineares a serem aplicados a situações onde exista a busca por soluções inovadoras. A partir dessa perspectiva, qual seria a melhor forma de atuar nessa questão?

As pesquisas realizadas a partir do início dos anos 1970 sobre os esforços organizacionais para inovar apontam npara a direção da composição do grupo como o principal fator de sucesso em relação à inovação (FLORIDA, 2003; VAN KNIPPENBERG; DE DREU; HOMAN, 2004; WOOLLEY et al., 2010). Organizações e produtos considerados retrospectivamente inovadores, surgiram de grupos que adotaram as mais diversas estratégias e técnicas, nos mais variados contextos, em situações de extrema escassez ou abundância de recursos. Ou seja, por um lado, não é possível apontar algum processo ou contexto decisivo para a criação de soluções inovadoras. Por outro, é possível perceber que tais grupos apresentaram uma certa flexibilidade de julgamentos que os capacitaram a sentir indícios e a se adaptar mais rapidamente às circunstâncias de contexto.

A análise destses grupos permite revelar que eles eram ou são formados por diferentes perfis de pessoas e regidos por um tipo de governança que promove a horizontalidade e a inclusão. Desta forma, as pesquisas sugerem uma perspectiva de aumento da produtividade das organizações em relação aos esforços de inovação, através de duas premissas básicas:

a) de que o respeito e a utilização efetiva das diferenças individuais gera ganhos de produtividade;

b)de que é possível habilitar grupos sociais a agir de forma a gerar propostas inovadoras sem a necessidade de métodos lineares.

Ou seja, ao invés de propor um método linear e de redução de diversidades para a geração de propostas inovadoras por grupos sociais, as pesquisas sugerem que o emprego efetivo da diversidade de preconceitos dos indivíduos em um grupo é um fator determinante para o aumento do potencial inovador desse mesmo grupo (MANHÃES, 2015).

\section{O TRIPLO DESAFIO DA INOVATIVIDADE ${ }^{1}$}

De maneira geral, as pesquisas (HONG; PAGE, 2004; ØSTERGAARD; TIMMERMANS; KRISTINSSON, 2011; VERGANTI; ÖBERG, 2013) apontam para o fato de que, para 
aumentar seus potenciais de gerar produtos considerados mais inovativos, os grupos sociais precisam vencer um desafio triplo. Esse triplo desafio exige que os grupos consigam: (i) entender os preconceitos de seus membros (GADAMER, 2004), (ii) entender o contexto social e histórico no qual esses grupos estão inseridos e, por fim, (iii) criar propostas inovativas que aumentem o repertório do possível (WEICK, 1995). Ou seja, focar apenas na "criação de propostas inovadoras" gera o ponto cego que fará reduzir o potencial inovador do grupo.

Para enfrentar tais desafios, para facilitar o compromisso de agir no sentido de enfrentar o triplo desafio da inovatividade, foi realizada uma pesquisa de doutorado junto ao Programa de Pós-gGradução em Engenharia e Gestão do Conhecimento da Universidade Federal de Santa Catarina - UFSC (MANHÃES, 2015). Como um dos resultados desta pesquisa, de abordagem interdisciplinar a respeito dos esforços de inovação de grupos, é proposta uma heurística denominada Prejudice Related Innovativeness Determinants Heuristic - PRIDHe. Ao mesmo tempo que esta proposição mantém o potencial de inovação das equipes, sem depender de processos de controle, ela permite que as organizações possam agir, fornecendo um discurso fundamentado academicamente sob a forma de uma heurística.

Figura 1 - Heuristica PRIDHe

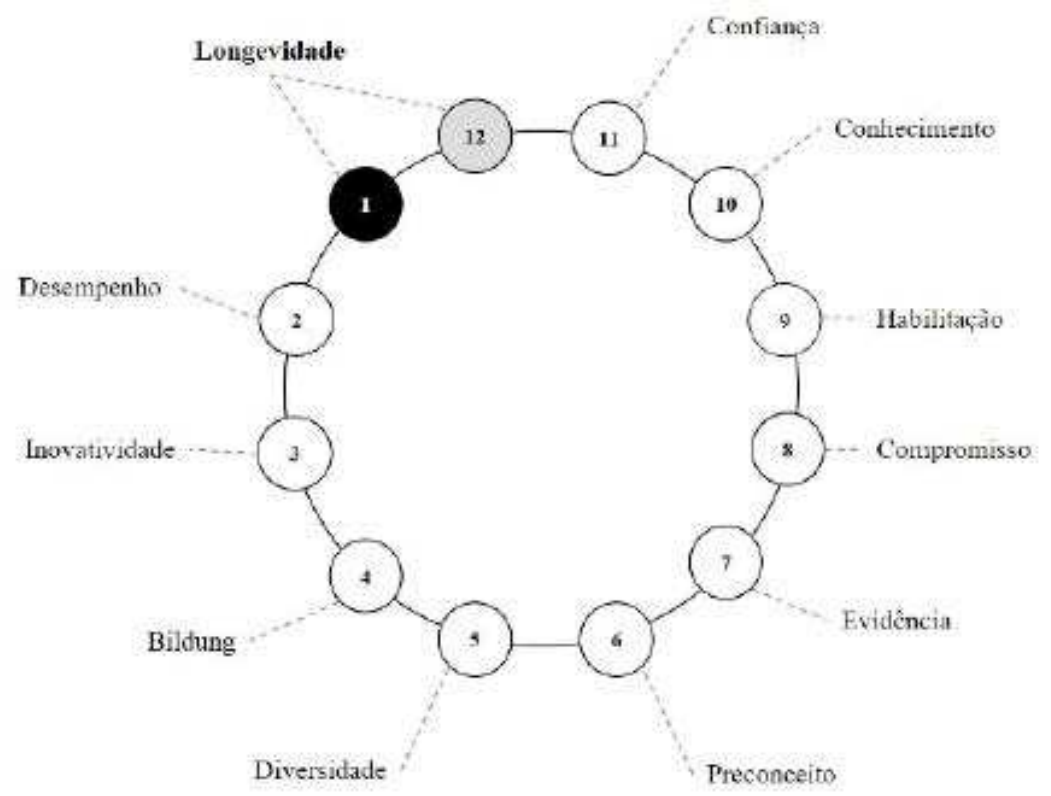

O discurso que suporta a heurística proposta é estruturado conforme a Figura 1, baseado em doze determinantes:

1) Quando as pessoas se sentem confiantes em um grupo, elas querem que ele seja longevo;

2) Para ser longevo, um grupo precisa ter um bom desempenho; 
3) Para ter um bom desempenho, um grupo precisa inovar;

4) Para inovar, um grupo tem que passar por um processo de Bildung ${ }^{2}$;

5) Para passar por um processo de Bildung, um grupo precisa dos benefícios da diversidade soócio-cultural;

6) Para obter os benefícios da diversidade soócio-cultural, um grupo tem que estar ciente dos preconceitos de seus membros;

7) Para estar ciente dos preconceitos de seus membros, um grupo precisa obter evidências;

8) Para obter evidências, um grupo precisa estar comprometido a agir;

9) Para se comprometer a agir, um grupo tem que ser habilitado para agir;

10) Para estar habilitado a agir, um grupo tem que criar novos conhecimentos;

11) Para criar novos conhecimentos, os membros do grupo precisam se sentir confiantes;

12) Ao se sentirem confiantes em um grupo, seus membros vão querer que ele seja longevo.

Enquanto o determinante 1 é uma atuação em direção ao futuro, o determinante 12 é um fazer sentido retrospectivamente (WEICK, 1995), ou seja, faz referência ao passado. Afinal de contas, o interesse na longevidade de um grupo surge em retrospecto, devido a explicações plausíveis sobre o que ocorreu ou está ocorrendo com as pessoas dentro de um grupo ou organização particular.

A operacionalização da heurística proposta para enfrentar o triplo desafio descrito é dividida em simples etapas, tais como:

1. Avaliação dos tipos de mentalidades (mente aberta e fechada): Com base nos resultados das pesquisas mencionadas e realizada pela referida tese (MANHÃES, 2015), é possível avaliar participantes de um grupo existente para verificar os níveis de abertura e fechamento de mente de cada indivíduo e, se necessário, proceder à seleção de pessoal ou realocação. Essa avaliação é obtida através da escala NFC (ROETS; VAN HIEL, 2011), que é um instrumento academicamente validado para medir diferentes tipos de mentalidade dos indivíduos. A escala NFC foi desenvolvida pelo Professor Arie W. Kruglanski (KRUGLANSKI, 2004) e é composta por 41 perguntas de onde são obtidos os níveis de NFC de cada indivíduo;

2. Design de Grupos Inovativos: Criação de grupos com base em uma combinação de indivíduos com diversos níveis de NFC. Trata-se de um processo simples para avaliar possíveis candidatos com a escala de NFC e conceber grupos com potenciais inovativos maiores com base nos níveis médios agregados de NFC. De acordo com a pesquisa doutoral mencionada, a capacidade de inovação dos grupos foi maior quando eles eram caracterizados por níveis médios de NFC entre 52 e 59 (quando consideradas as respostas para as 41 questões colocadas acima) e quando possuíam um Coeficiente de Variação entre 0,14 e 0,24 (MANHÃES, 2015). 
As diferenças entre os grupos que estão nessa faixa de NFC e aqueles que não estão sugere que aqueles obtêm uma avaliação quase $50 \%$ superior quanto ao nível de percepção de inovatividade dos seus produtos;

3. Políticas de Governança: Adoção de políticas de governança que impõem o contato intergrupal não-hierárquico. Por exemplo, se a organização já avaliou seus membros para formar um grupo com alto potencial inovativo, ela deve em seguida assegurar que este grupo obedece à uma política de governança adequada que irá reforçar o seu potencial inovativo. Portanto, a adoção de uma política de governança que promove a consciência dos preconceitos que atuam em determinado contexto pode ser considerada como uma estratégia de reforço da capacidade inovativa de um grupo. A política de governança sugerida deve ser baseada nas condições-chave de contato intergrupal desenvolvidas pelo Professor Gordon Allport (ALLPORT, 1979), sendo elas: (i) criar condições de igualdade de status entre indivíduos ou grupos; (ii) necessariamente dirigir todas as ações individuais em prol de objetivos comuns; (iii) objetivos que só podem ser atingidos através da cooperação e interdependência obrigatórias; e (iv) as condições anteriores devem ser apoiadas por mensagens e ações claras das autoridades quanto à obediência a elas (PETTIGREW, 1998);

4. Autonomia: Proporcionar autonomia organizacional para os grupos formados. A organização não necessita obrigatoriamente ter uma ideia clara de como ela vai "atuar no futuro." Portanto, o melhor lugar para começar a agir no futuro é procurar evidências de que os seus membros formam um grupo de alto potencial inovativo e, em seguida, assegurar que esse grupo obedece à uma política de governança adequada e que irá favorecer o seu potencial inovativo. Por fim, a organização deve fornece autonomia para o grupo inovativo e pedir aos membros desse grupo que eles próprios definam maneiras de "atuar na direção do futuro" (LUDZ, 1999), criando autonomamente cursos de ação, metas, objetivos e resultados;

5. Recursos: Definição de prazos e recursos disponíveis, acompanhar a aplicação desses recursos e verificar continuamente se o grupo obedece à governança adotada e está comprometido com o processo de inovatividade, com as suas metas e resultados, tanto para o próprio grupo quanto para a organização como um todo.

As etapas sugeridas acima devem permitir que as organizações possam criar grupos propensos ao processo de Bildung (GADAMER, 2004) nos quais a imaginação produtiva é mais rica, porque não vai ser apenas "livre." Os horizontes de possiblidades descortinados por esses grupos não-livres, "tal como nas convoluções dos arabescos," deve prover um espaço de jogo no qual o desejo por unidade (padrão) estabelecido por todo esforço de compreensão "não apenas confina como também sugere incitamentos ao jogo" (GADAMER, 2004, p. 41). A pesquisa doutoral 
mencionada demonstra que a adoção de práticas que aumentam a consciência dos preconceitos em ação dentro de determinado contexto promove a confiança dos membros e das comunidades vizinhas ao grupo. Se a organização não tem uma ideia clara de como ela pretende "atuar na direção do futuro" e nem sabe como/onde é o melhor lugar para "compreender a realidade", seus administradores devem assegurar que o primeiro passo se dê com a adoção de uma política de governança adequada e que irá promover a sua inovatividade potencial. Tal governança deve promover a construção de pontes para permitir a organização conectar-se com Outros; ou seja, com diferentes discursos e realidades.

\section{VERIFICAÇÃO DO POTENCIAL INOVATIVO}

A partir da heurística proposta, é possível sugerir uma Verificação do Potencial Inovativo para as organizações. Esta análise foi criada para ser usada por gestores organizacionais, a fim de verificar se a respectiva organização tem o potencial necessário para enfrentar o triplo desafio de inovatividade. O processo de análise proposto é baseado em cinco perguntas, para as quais os gestores precisam responder "Sim" ou "Não." As perguntas são:

1. Os membros da organização, como um todo, representam diversos tipos de mentalidades (mente aberta e fechada)?

2. O grupo diretamente responsável pelo esforço inovativo é composto por uma mescla ideal de indivíduos com diferentes mentalidades (mente aberta e fechada)?

3. Esse grupo obedece à uma política de governança que impõe condições específicas de contato entre indivíduos e grupos, sendo elas: (a) igualdade de status em todas situações; (b) estabelecimento apenas de metas compartilhadas e (c) atividades calcadas na interdependência profunda entre os membros?

4. O referido grupo tem total autonomia para definir cursos de ação, metas, objetivos e resultados?

5. O grupo diretamente responsável pelo esforço inovativo foi claramente informado sobre os recursos (orçamento e prazo) que será obrigado a cumprir?

Estas questões estão diretamente relacionadas aos cinco itens descritos anteriormente para a operacionalização simplificada da heurística proposta. Para aumentar a probabilidade de enfrentar com sucesso o triplo desafio da inovatividade, com base no raciocínio que apoia a presente heurística, os gestores das organizações têm de responder positivamente (sim) para todas as questões apresentadas acima. Cada resposta negativa solicita aos gestores implementar as diretrizes sugeridas pelo item correspondente, a partir dos cinco itens previamente descritos.

Os resultados da pesquisa mencionada (MANHÃES, 2015) confirmam que 
o nível médio de NFC de grupos (níveis esses, gerados a partir do ponto de vista histórico de seus membros) impactam os resultados dos esforços inovativos desses mesmos grupos. $E$, também, que é possível a composição de grupos com maior potencial inovativo. A abordagem interdisciplinar realizada pela pesquisa a respeito dos esforços inovativos realizados por grupos contribui para diminuir o ponto cego - representado pelas tradições e preconceitos de um grupo - nos esforços organizacionais em relação à inovação. Afinal, ao mesmo tempo em que esta abordagem mantém o potencial de inovação das equipes - sem depender de processos de controle e linearidade, ela permite que as organizações atuem de forma produtiva, fornecendo um discurso academicamente suportado na forma de uma heurística.

\section{NOTAS}

[1] Este texto apoia-se na conceituação de que o fenômeno da inovação é de difícil previsibilidade e só pode ser efetivamente avaliado após decorrido considerável tempo, na medida em que ele "desdobra-se através de décadas ou séculos" (SCHUMPETER, 1943). Desta feita, para os propósitos deste texto, é adotado o conceito de inovatividade como uma medida do grau de "novidade" que é percebido por um determinado grupo social a respeito de um produto específico. Portanto, o grau de inovatividade de determinado produto está intrínsicamente relacionado à "perspectiva de novidade de quem o percebe" e o que é considerado como novidade por quem o avalia (GARCIA; CALANTONE, 2002).

[2] Em Português, Bildung corresponde à "formação" e pode ser entendida como o esforço de "manter-se aberto para o que é outro - para outros e mais universais pontos de vista" (GADAMER, 2004, p. 15), o que pode ser considerado uma condição fundamental para os esforços de cocriação, especialmente para a obtenção de propostas inovadoras.

\section{BIBLIOGRAFIA}

ADORNO, T. Functionalism today. Rethinking Architecture: A reader in cultural theory, p. 1-12, 1965.

ALLPORT, G. The Nature of Prejudice: 25th Anniversary Edition. [s.I.] Perseus Books Publishing, L.L.C., 1979.

AMABILE, T. M. Creativity and innovation in organizations. [s.l.] Harvard Business School, 1996. 
BUCHANAN, R. Worlds in the Making: Design, Management, and the Reform of Organizational Culture. She Ji: The Journal of Design, Economics, and Innovation, set. 2015.

FLORIDA, R. Cities and the creative class. City \& Community, v. 2, n. 1, p. 3-19, 2003.

GADAMER, H.-G. Truth and Method. Second Edi ed. London: Continuum, 2004.

GARCIA, R.; CALANTONE, R. A critical look at technological innovation typology and innovativeness terminology: a literature review. Journal of Product Innovation Management, v. 19, n. 2, p. 110-132, mar. 2002.

GINOULHIAC, M. O ensino do projecto de Arquitectura Contribuições para um debate crítico em torno da prática contemporânea. [s.I.] UNIVERSIDADE DO PORTO, 2009.

HONG, L.; PAGE, S. E. Groups of diverse problem solvers can outperform groups of high-ability problem solvers. Proceedings of the National Academy of Sciences of the United States of America, v. 101, n. 46, p. 16385-9, 16 nov. 2004.

JOHANSSON-SKÖLDBERG, U.; WOODILLA, J.; ÇETINKAYA, M. Design Thinking: Past, Present and Possible Futures. Creativity and Innovation Management, v. 22, n. 2, p. 121-146, 25 jun. 2013.

KRUGLANSKI, A. W. The Psychology of Closed Mindedness (Essays in Social Psychology). New York: Psychology Press, 2004.

LUDZ, U. Interviewing Hannah Arendt. Hannah Arendt Newsletter, 1999.

MANHÃES, M. C. INNOVATIVENESS AND PREJUDICE: DESIGNING A LANDSCAPE OF DIVERSITY FOR KNOWLEDGE CREATION. [s.I.] Universidade Federal de Santa Catarina, Centro Tecnológico, 2015.

ØSTERGAARD, C. R.; TIMMERMANS, B.; KRISTINSSON, K. Does a different view create something new? The effect of employee diversity on innovation. Research Policy, v. 40, n. 3, p. 500-509, abr. 2011.

PETTIGREW, T. F. Intergroup contact theory. Annual review of psychology, v. 49, p. 65-85, jan. 1998.

ROETS, A.; VAN HIEL, A. Item selection and validation of a brief, 15-item version of the Need for Closure Scale. Personality and Individual Differences, v. 50, n. 1, p. 90-94, jan. 2011.

SCHUMPETER, J. A. The Explanation of the Business Cycle. Economica, v. 21, n. 21, p. 286-311, 1927.

SCHUMPETER, J. A. Capitalism, socialism and democracy. London: Routledge, 1943.

SEWELL, W. H. The temporalities of capitalism. Socio-Economic Review, v. 6, n. 3, p. 517-537, 15 abr. 2008.

SIMONTON, D. K. Creative thought as blind-variation and selective-retention: 
combinatorial models of exceptional creativity. Physics of life reviews, v. 7, n. 2, p. 156-179, jun. 2010.

TANGEN, S. Demystifying productivity and performance. International Journal of Productivity and Performance Management, v. 54, n. 1, p. 34-46, 2005.

THOMPSON, C. Interpreting Consumers: A Hermeneutical Framework for Deriving Marketing Insights from the Texts of Consumers' Consumption Stories. Journal of marketing Research, v. 34, n. 4, p. 438-455, 1997.

VAN KNIPPENBERG, D.; DE DREU, C. K. W.; HOMAN, A. C. Work group diversity and group performance: an integrative model and research agenda. The Journal of applied psychology, v. 89, n. 6, p. 1008-22, dez. 2004.

VERGANTI, R.; ÖBERG, Å. Interpreting and envisioning - A hermeneutic framework to look at radical innovation of meanings. Industrial Marketing Management, v. 42, n. 1, p. 86-95, jan. 2013.

WEICK, K. E. Sensemaking in Organizations. London: Sage Publications, 1995.

WOOLLEY, A. W. et al. Evidence for a collective intelligence factor in the performance of human groups. Science (New York, N.Y.), v. 330, n. October, p. 686-688, 2010.

Recebido 12/04/2016; Aceito em: 21/04/2015.

Esta obra foi licenciada com uma Licença Creative Commons.
Gregório Varvakis é Doutor em Manufacturing Engineering pela Loughborough University of Technology, Inglaterra e Professor titular do Departamento de Engenharia e Gestão do Conhecimento da Universidade Federal de Santa Catarina.

Maurício Manhães possui Doutorado em Engenharia e Gestão do Conhecimento (UFSC), com foco no impacto do preconceito nos esforços de inovação. Atualmente, é Professor de Design de Serviço na Savannah College of Art and Design (SCAD), na Georgia/EUA. Possui sólida experiência em design de interfaces e gestão de projetos de TI. Desde 2008, foca sua atuação na pesquisa e desenvolvimento da inovação em serviços através do design.

Tarcisio Vanzin é Doutor em Engenharia de Produção pela Universidade Federal de Santa Catarina, Brasil e Professor voluntário do Programa de Pós Graduação em Arquitetura e Urbanismo e do Programa de Pós Graduação em Engenharia e Gestão do Conhecimento da Universidade Federal de Santa Catarina 